\title{
Effects of statins on serum polyunsaturated fatty acids
}

\author{
Satoshi Kurisu
}

Received: 12 March 2012/Accepted: 3 April 2012/Published online: 6 June 2012

(C) Springer 2012

I thank Dr. Berent and Dr. Sinzinger for thoughtful comments and appreciate their great interest in our study. Because baseline low-density lipoprotein (LDL) cholesterol was too high to achieve a level less than $100 \mathrm{mg} / \mathrm{dl}$ using low-potency statins, we used high-potency statins including atorvastatin, rosuvastatin, or pitavastatin for lipid-lowering therapy. Consequently, LDL cholesterol less than $100 \mathrm{mg} / \mathrm{dl}$ was achieved in $65 \%$ of the patients. These high-potency statins are often called "strong statins" in the clinical setting [1, 2]. Because we used only highpotency statins in our study, the manuscript was entitled effects of lipid lowering therapy with "strong statin" on polyunsaturated fatty acid acids (PUFAs). I do not argue that only strong statins have the effects reducing n-3 PUFAs in proportion to the decrease in the LDL cholesterol. I agree with your suggestion that a higher dose of lowpotency statins may have the same effects. However, a previous study by Nakamura et al. [3] showed that lowpotency statins (pravastatin and simvastatin) decreased LDL cholesterol from 159 to $117 \mathrm{mg} / \mathrm{dl}$, and increased arachidonic acid (AA) with no significant effects on eicosapentaenoic acid (EPA) and docosahexaenoic acid (DHA). On the other hand, we demonstrated that highpotency statins decreased LDL cholesterol from 131 to $89 \mathrm{mg} / \mathrm{dl}$, and decreased EPA and DHA with no significant effect on AA. There was an inconsistency of the results between the previous study using low-potency statins and

This author's reply refers to the letter to the editor at doi:10.1007/s00380-012-0255-4.

\section{S. Kurisu $(\square)$}

Department of Cardiovascular Medicine, Hiroshima University

Graduate School of Biomedical Sciences, 1-2-3 Kasumi-cho,

Minami-ku, Hiroshima 734-8551, Japan

e-mail: skurisu@nifty.com our study using high-potency statins. Further studies are necessary to clarify whether the inconsistency results from the extent of LDL cholesterol lowering or the potency of statins.

There was no wash out phase before the change from a low-potency statin to a high-potency statin. The presence of a wash out phase is helpful to clarify the effects of strong statins on PUFAs. However, it is not ethical in patients with coronary artery disease. This was why we did not make a wash out phase in our study.

Statins cause stabilization and regression of plaque, as well as inhibiting adverse cardiovascular events through only LDL cholesterol lowering but also several pleiotropic effects. Statins are established drugs that should be prescribed in all patients with coronary artery disease regardless of LDL cholesterol level. The serious problem is the recurrence of adverse cardiovascular events in patients receiving statin therapy. I think that additional treatment with n-3 PUFAs may be a promising strategy especially in patients with a high risk of coronary artery disease.

\section{References}

1. Ogata N, Fujimori S, Oka Y, Kaneko K (2010) Effects of three strong statins (atorvastatin, pitavastatin, and rosuvastatin) on serum uric acid levels in dyslipidemic patients. Nucleosides Nucleotides Nucleic Acids 29:321-324

2. Ikewaki K, Ayaori M (2011) Strong statins as the major players for dyslipidemia in high-risk patients: are they all the same or not? Circ J 75:1326-1327

3. Nakamura N, Hamazaki T, Jokai H, Minami S, Kobayashi M (1998) Effect of HMG-CoA reductase inhibitors on plasma polyunsaturated fatty acid concentrations in patients with hyperlipidemia. Int J Clin Lab Res 28:192-195 